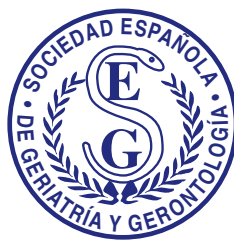

\title{
Revista Española de Geriatría y Gerontología
}

\section{CARTA CIENTÍFICA}

\section{Neumonía adquirida en la comunidad en un hospital terciario español: ¿cómo se comporta en pacientes provenientes de residencia?}

\section{Community acquired pneumonia in a Spanish tertiary hospital: how does it work in patients from residence?}

Con 4,63 casos por cada 1.000 personas/año ${ }^{1}$ y 1,64 hospitalizaciones por cada 1.000 personas/año ${ }^{2}$ la neumonía adquirida en la comunidad (NAC) es una enfermedad frecuente en España. Varias guías de práctica clínica resumen el abordaje más adecuado para su manejo ${ }^{3-5}$. Sin embargo, existe cierto debate sobre cómo se comportan los pacientes provenientes de residencia ${ }^{3,6}$. En el presente artículo analizamos las características de un grupo de pacientes hospitalizados por NAC, comparando aquellos provenientes de residencia con los que no.

Se recogieron retrospectivamente todos los casos de NAC durante 9 meses (1/1/16 al 31/9/16) en el Servicio de Medicina Interna (SMI) del Hospital Clínico Universitario Lozano Blesa de Zaragoza. Se reclutaron los pacientes mayores de 18 años diagnosticados de NAC por su médico, excluyendo la neumonía nosocomial (aquella que se presenta tras $48 \mathrm{~h}$ del ingreso o en los 7 días tras el alta) y la infección respiratoria sin neumonía. Para ello se revisaron

Tabla 1

Comparación entre pacientes con NAC provenientes de residencia frente al resto

\begin{tabular}{|c|c|c|c|c|}
\hline NAC en pacientes provenientes de residencia & Sí $(\mathrm{n}=119)$ & No $(n=194)$ & Valor de $\mathrm{p}$ & Global $(n=313)$ \\
\hline Edad en años; media & $84,3(8,7)$ & $79,3(12,6)$ & $<0,001$ & $81,2(11,5)$ \\
\hline Sexo mujer; $n(\%)$ & $59(49,6)$ & $82(42,3)$ & 0,207 & $141(45)$ \\
\hline Índice de Charlson; media (DT) & $6(2,6)$ & $5,1(3)$ & 0,005 & $5,4(2,9)$ \\
\hline Demencia; n (\%) & $72(60,5)$ & $43(22,2)$ & $<0,001$ & $115(36,7)$ \\
\hline Diabetes mellitus; n (\%) & $30(25,2)$ & $55(28,4)$ & 0,544 & $85(27,2)$ \\
\hline Enfermedad cerebrovascular; n (\%) & $31(26,1)$ & $41(21,1)$ & 0,316 & $72(23)$ \\
\hline EPOC; $\mathrm{n}(\%)$ & $23(19,3)$ & $45(23,2)$ & 0,420 & $68(21,7)$ \\
\hline Enfermedad renal crónica; n (\%) & $20(16,8)$ & $38(19,6)$ & 0,539 & $58(18,5)$ \\
\hline Insuficiencia cardíaca; n (\%) & $20(16,8)$ & $34(17,5)$ & 0,870 & $54(17,3)$ \\
\hline \multicolumn{5}{|l|}{ Síntomas } \\
\hline Disnea; n (\%) & $78(65,5)$ & $119(65,5)$ & 0,455 & $197(62,9)$ \\
\hline Tos; $\mathrm{n}(\%)$ & $53(44,5)$ & $131(67,5)$ & $<0,001$ & $184(58,8)$ \\
\hline Fiebre; n (\%) & $70(58,8)$ & $109(56,2)$ & 0,647 & $179(57,2)$ \\
\hline Expectoración; n (\%) & $38(31,9)$ & $92(47,4)$ & 0,007 & $130(41,5)$ \\
\hline Escalofríos; n (\%) & $2(1,7)$ & $16(8,2)$ & 0,015 & $18(5,8)$ \\
\hline Dolor torácico; n (\%) & $8(6,7)$ & $39(20,1)$ & 0,001 & $47(15)$ \\
\hline Artromialgias; $\mathrm{n}(\%)$ & 0 & $9(4,6)$ & 0,015 & $9(2,9)$ \\
\hline CURB-65; media (DT) & $2,7(1)$ & $2(1,1)$ & $<0,001$ & $2,2(1,1)$ \\
\hline SOFA; media $(n=208)(D T)$ & $3,9(2)$ & $2,9(1,6)$ & $<0,001$ & $3,3(1,8)$ \\
\hline PSI; media $(n=157)(D T)$ & $144(29,9)$ & $113(31)$ & $<0,001$ & $124(34,6)$ \\
\hline Intento de diagnóstico; $n(\%)$ & $98(82,4)$ & $184(94,8)$ & $<0,001$ & $282(90,1)$ \\
\hline$N .^{\circ}$ de pruebas microbiológicas solicitadas; media & $1,69(1,2)$ & $2,42(1,24)$ & $<0,001$ & $2,15(1,28)$ \\
\hline Hemocultivos; n (\%) & $57(47,9)$ & $111(57,2)$ & 0,109 & $168(53,7)$ \\
\hline Esputo; n (\%) & $27(22,7)$ & $105(54,1)$ & $<0,001$ & $132(42,2)$ \\
\hline Antigenuria; n (\%) & $79(66,4)$ & $155(79,9)$ & 0,008 & $234(74,8)$ \\
\hline Gripe; n (\%) & $4(3,4)$ & $29(14,9)$ & 0,001 & $33(10,5)$ \\
\hline Diagnóstico microbiológico; $n(\%)$ & $23(19,3)$ & $46(23,7)$ & 0,364 & $69(22)$ \\
\hline Aislamiento de P. aeruginosa o K. pneumoniae & $1(0,8)$ & $8(4,1)$ & 0,161 & $9(2,9)$ \\
\hline Duración del tratamiento antibiótico iv (días); media (DT) $(n=187)$ & $5,3(3,3)$ & $4,3(2,3)$ & 0,02 & $4,6(2,7)$ \\
\hline Duración de tratamiento total (días); media (DT) $(n=302)$ & $8,38(4,4)$ & $9,14(4)$ & 0,126 & $8,9(4,2)$ \\
\hline Uso de carbapenems o pipe/tazo durante parte o la totalidad del tratamiento; $n$ (\%) & $26(22,4)$ & $41(21,6)$ & 0,864 & $67(21,4)$ \\
\hline Estancia (días); media $(n=279)(D T)$ & $8,25(5)$ & $9,8(6,2)$ & 0,444 & $9,6(5,8)$ \\
\hline Reingreso a 30 días; $n(\%)$ & $16(13,4)$ & $17(8,8)$ & 0,19 & $33(10,5)$ \\
\hline Exitus intrahospitalario; $n(\%)$ & $18(15,1)$ & $16(8,2)$ & 0,058 & $34(10,9)$ \\
\hline Exitus $<30$ días del alta; $n(\%)$ & $39(32,8)$ & $24(12,4)$ & $<0,001$ & $63(21,1)$ \\
\hline
\end{tabular}

CURB65: escala confusión, urea frecuencia respiratoria presión arterial sistólica, edad; DT: desviación típica; EPOC: enfermedad pulmonar obstructiva crónica; iv: intravenoso;

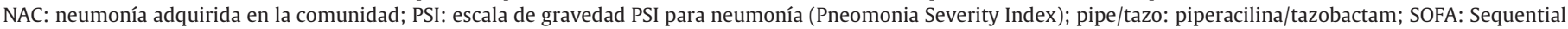
Organ Failure Assessment; Intento diagnóstico: se dio como «sí» si se solicitó al menos una prueba diagnóstica.

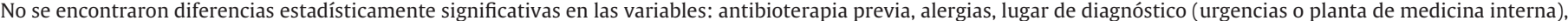
aislamiento de S. pneumoniae, complicaciones, serología, toma de muestra de líquido pleural. 
diariamente los ingresos y altas del SMI. Se consideró paciente con NAC proveniente de residencia si así se hacía constar en la historia clínica. Se registraron variables demográficas, clínicas, diagnósticas, terapéuticas y de resultados. Posteriormente se comparó el grupo de pacientes con NAC provenientes de residencia con el resto, empleando los test de Chi-cuadrado o la $t$ de Student y la razón de probabilidades (OR). Se consideró una $\mathrm{p}<0,05$ como estadísticamente significativa. Se empleó el programa estadístico SPSS ${ }^{\circledR}$ versión 15.

Reclutamos una muestra de 313 pacientes con una mediana de duración de tratamiento y estancia de 8 días. Sus características vienen detalladas en la tabla 1. En relación a la comparación entre pacientes que provenían de domicilio o residencia, encontramos que estos últimos fueron más añosos y tuvieron más comorbilidades, especialmente demencia (OR: 5,48; IC 95\%: 3,16-9,87; $\mathrm{p}<0,001)$. La presentación sintomática fue menos florida en ellos. También se realizaron menos pruebas diagnósticas, siendo más probable que no se intentara realizar diagnóstico microbiológico (OR: 3,94; IC 95\%: 1,79-8,7; p <0,001). Se empleó la vía intravenosa durante más tiempo, sin encontrar diferencias en cuanto a la duración total o a la estancia. La mortalidad en los pacientes institucionalizados fue mayor, con un OR: 3,45; IC 95\%: 1,95-6,12; $\mathrm{p}<0,001$ para la mortalidad total hasta 30 días del alta en dicho grupo (tabla 1 ).

Encontramos que el caso típico de NAC consiste en un paciente de avanzada edad y con un alto grado de comorbilidades, siendo más acusados estos hallazgos en los que provenían de residencia. No se encontró un mayor grado de infecciones por bacilos gramnegativos como P. aeruginosa o K. pneumoniae ni se usó más piperacilina/tazobactam o carbapenems en estos pacientes, aunque es necesario reseñar que se realizó menos intento de diagnóstico. Consideramos que esta menor obstinación diagnóstica pudo deberse a la presentación paucisintomática de las enfermedades en los pacientes con mayor edad y deterioro cognitivo, así como a que se hayan podido instaurar medidas de limitación del esfuerzo médico en ellos. En relación a ello, en la literatura se plantea que el manejo de la NAC en pacientes institucionalizados como si fueran neumonías nosocomiales podría conllevar un sobreuso de antibióticos de espectro excesivo, siendo importante el conocimiento de la epidemiología local ${ }^{3,6}$; y que el concepto de neumonía asociada a cuidados sanitarios se basa en estudios de poca calidad y que no identifica con precisión la probabilidad resistencia ${ }^{7}$. Respecto a la mayor mortalidad del grupo de pacientes provenientes de residencia, creemos que esta pueda deberse tanto a sus características basales (edad, comorbilidades), como a que se hayan llevado a cabo medidas de limitación del esfuerzo terapéutico, dato este último que no ha sido recogido. El carácter observacional de nuestro trabajo hace que se preste a todos los sesgos de este tipo de estudios, siendo especialmente vulnerable a la posible pérdida de información en el caso de que determinados datos no se recogieran en la historia. Sin embargo, creemos haber retratado el perfil típico de paciente de nuestro medio. En esencia, la sociedad en nuestros días se enfrenta al reto de la longevidad, la dependencia y las enfermedades neurodegenerativas; y las personas institucionalizadas son el ejemplo de estas características. En este sentido, se hace necesario conocer bien el comportamiento de este tipo de pacientes para brindarles el manejo óptimo que mejore la carga asistencial, el uso de antibióticos y su calidad de vida. Abordar asuntos relacionados con la limitación del esfuerzo terapéutico y el manejo ambulatorio en estos pacientes podría perfilarse como una aproximación a esta estrategia en casos seleccionados ${ }^{8}$.

\section{Financiación}

Los autores declaran no haber recibido financiación alguna para este trabajo.

\section{Bibliografía}

1. Rivero-Calle I, Pardo-Seco J, Aldaz P, Vargas DA, Mascarós E, Redondo E, et al., NEUMOEXPERTOS group. Incidence and risk factor prevalence of community-acquired pneumonia in adults in primary care in Spain (NEUMO-ES-RISK project). BMC Infect Dis. 2016;16:645.

2. de Miguel-Díez J, Jiménez-García R, Hernández-Barrera V, Jiménez-Trujillo I de Miguel-Yanes JM, Méndez-Bailón M, et al. Trends in hospitalizations for community-acquired pneumonia in Spain: 2004 to 2013. Eur J Intern Med. 2017;40:64-71.

3. Mandell LA, Wunderink RG, Anzueto A, Bartlett JG, Campbell GD, Dean NC, et al., Infectious Diseases Society of America; American Thoracic Society. Infectious Diseases Society of America/American Thoracic Society Consensus Guidelines on the Management of Community-Acquired Pneumonia in Adults. Clin Infect Dis. 2007:44 Suppl 2:S27-72.

4. Lim WS, Baudouin SV, George RC, Hill AT, Jamieson C, Le Jeune I, et al., Pneumonia Guidelines Committee of the BTS Standards of Care Committee. BTS guidelines for the management of community acquired pneumonia in adults: Update 2009. Thorax. 2009;64 Suppl 3:iii1-55.

5. Woodhead M, Blasi F, Ewig S, Garau J, Huchon G, Ieven M, et al. Guidelines for the management of adult lower respiratory tract infections - Summary. Clin Microbio Infect. 2011;17 Suppl 6:1-24.

6. Wunderink RG, Waterer G. Advances in the causes and management of community acquired pneumonia in adults. BMJ. 2017;358:2471.

7. Chalmers JD, Rother C, Salih W, Ewig S. Healthcare-associated pneumonia does not accurately identify potentially resistant pathogens: A systematic review and meta-analysis. Clin Infect Dis. 2014;58:330-9.

8. González-Castillo J, Martín- FJ, Mujal A, Navas E. Consensus document Guidelines for the management of community-acquired pneumonia in the elderly patient. Rev Esp Quim. 2014;27:69-86.

David Sánchez Fabra ${ }^{a}{ }^{*}$, Fernando J. Ruiz Laiglesia ${ }^{a}$, Galadriel Pellejero Sagastizabal ${ }^{a}$,

María José Cumbraos Sánchez ${ }^{\mathrm{b}}$, Susana Olivera González ${ }^{\mathrm{a}}$, María Ángeles Allende Bandrés ${ }^{\mathrm{b}}$, Araceli Jimeno Sainz ${ }^{\mathrm{a}}$, Carmina Vicente de Vera ${ }^{a}$, María Asunción Sagredo Samanes ${ }^{\mathrm{b}}$ y José Ramón Paño Pardo ${ }^{c}$

a Servicio de Medicina Interna, Hospital Clínico Universitario Lozano Blesa, Zaragoza, España

b Servicio de Farmacia Hospitalaria, Hospital Clínico Universitario Lozano Blesa, Zaragoza, España

c Servicio de Enfermedades Infecciosas, Hospital Clínico Universitario Lozano Blesa, Zaragoza, España

* Autor para correspondencia.

Correo electrónico: davidsanchezfabra@gmail.com (D. Sánchez Fabra) 